ISSN 1991- 8690

website : http:// jsci.utq.edu.iq

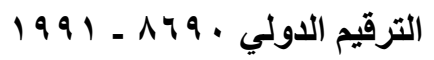

Email: utjsci@utq.edu.iq

\title{
Theoretical Study of the Effect of the Coupling Constant Strength on the Photons Rate Yield for Quark Gluon Interaction
}

\author{
Hadi J. M. AL-Agealy ${ }^{1}$
}

\author{
Ahmed Aziz ${ }^{2}$
}

Mudhafar J. Sahib ${ }^{1}$

${ }^{1}$ Department of Physics- College of Education for Pure Science -Ibn Al- Haitham-University of Baghdad.

${ }^{2}$ Department of Physics- College of Science- The University of Mustansiriyah.

Keywords: theoretical study, coupling constant strength, Photons Rate Yield, quark- gluon interaction

\begin{abstract}
$\underline{\text { Abstract }}$
Rate of photon yield in a quark-gluon interaction exhibits of the coupling constant strength and temperature dependence have been studied theoretically. The estimation of the coupling constant strength is very important tool for the investigation of the QCD, which has been applied to the dynamical quarks $n_{f}=3$, 5, and gluon $n_{f}=0$. Depending upon the the coupling constant data, it shows that the strong force is relatively weak for quarks, and the two quarks move farther apart the force becomes stronger. The weakness of interaction at short distance is called asymptotic freedom, while the strength of the long distance is called confinement .Rate of the photon yield in a quark-gluon interaction which investigation due to the electric charge of the quark with color quantum number. Photon's rate has been calculated increasing with increases in temperature and decreasing the coupling constant and photon's yield from the system has height fever number larger than system has low fever number .
\end{abstract}

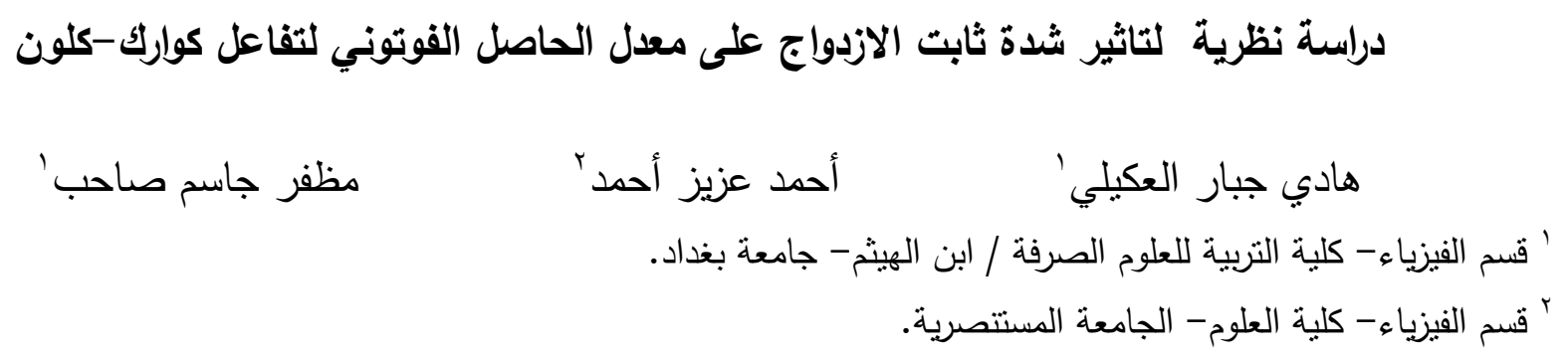

الماخص

معدل الحاصل الفوتوني في عملية تفاعل الكوارك - كلوون درست نظريا وفقا لثابت الازدواج ودرجة الحرارة .حساب شدة ثابت الازدواج هو اداة مهمة لفهم النظرية الكمية اللونية والتي تفسر حركية الكوارك ـ اعتمادا على نتائج ثابت شدة الازدواج كنرى ان القوى القورية القورية تضعف للكواركات ولكواركين

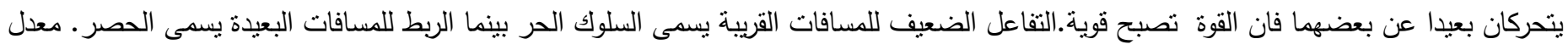

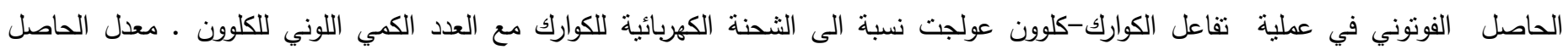

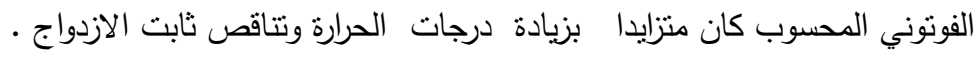
الكلمات المفتاحية: دراسة نظرية، شدة ثابت الاقتران، معدل الحاصل الفوتوني، تفاعل كوارك-كلون. 


\section{1- Introduction}

Over the past twenty years, one of the most important aims in the heavy-ion experiments is to explore a new form of matter (the quark-gluon interaction) which has been predicted by the quantum chromodynamics at finite temperature [1]. Quantum Chromodynamics is a field theory which describes the strong interaction between the quarks and gluons. It is generally assumed that all fundamental particles (among them quarks and gluons) are represented by local quantum fields. In particle physics there is a consensus to describe all local fields by quantum field theories, and QCD is one such theory. QCD, in its attempt to describe the strong interactions, introduces the concept of quarks and colour. The quark fields represent the matter fields, and although they are not directly observable, they form hadrons which are observed in nature[2]. In 1964 quarks were introduced independently by Gell-Mann, and Zweig as building blocks of hadronic matter. As far as we know today, quarks are indivisible elementary particles. Hadrons are divided into two groups. One group called baryons, consisting of three quarks (qqq) like protons and neutrons. The other group called mesons, consisting of a quark-antiquark pair $(q q)$ like pions and kaons[3]. In the standard model of quark gluon interaction, there are six quarkfavors $\mathrm{u} ; \mathrm{d} ; \mathrm{s} ; \mathrm{c} ; \mathrm{t}$ and $\mathrm{b}$ and three quark colors $\mathrm{R} ; \mathrm{W}$; and $\mathrm{B}$. The quarks are matter fields. The potential of the radiated gluon field also has SU (3) symmetry, and in the standard model the various gluons are considered to be a massless particles[4]. Table(1) show some properties of quarks [5].

Table (1): Properties of quark flavors, their relative electric charges and masses [5].

\begin{tabular}{|c|c|c|}
\hline \multicolumn{3}{|c|}{ Quarks } \\
\hline Quark Flavor & Electric Charge & Mass \\
\hline U (up) & $+2 / 3 e$ & 1.5 to $3.3 \mathrm{MeV} / c^{2}$ \\
\hline D (down) & $-1 / 3 e$ & 3.5 to $6.0 \mathrm{MeV} / c^{2}$ \\
\hline C (charm) & $+2 / 3 e$ & $1.27_{-0.11}^{+0.07} \mathrm{GeV} / c^{2}$ \\
\hline S (strange) & $-1 / 3 e$ & $104_{-34}^{+26} \mathrm{MeV} / c^{2}$ \\
\hline T (top) & $+2 / 3 e$ & $171.2 \pm 2.1 \mathrm{GeV} / c^{2}$ \\
\hline B (bottom) & $-1 / 3 e$ & $4.20_{-0.07}^{+0.17} \mathrm{GeV} / c^{2}$ \\
\hline
\end{tabular}

Photons are produced mainly in charged particle scattering. The production of direct photons was predicted as a good signature for the study of a quark gluon [6].The term "direct photon" refers to those photons which are produced in the hard-scattering subprocess and are not decay products of some particle. photons can only interact electromagnetically and so any photons produced in the quark-gluon phase are likely to survive until detected. The dominant source of photon production in a quark-gluon was originally thought to be $\mathrm{gq} \rightarrow \gamma \mathrm{q}$ (with a small contribution from $\mathrm{q} \overline{\mathrm{q}} \rightarrow \gamma \mathrm{g}$ ), and that of a thermal. The result of which was that photon production rates in a quark-gluon and hadrons gas were thought to be similar: i.e. photon production was dependent on temperature, but not necessarily a signature of a quark-gluon[7]. The strength of the quark-gluon interaction, characterized by the strong coupling constant $\alpha_{s}$. As a consequence, quarks can only exist in bound states, a property known as color confinement. For large momentum transfer, it is possible to calculate QCD processes accurately using a perturbative expansion in $\alpha_{\mathrm{s}}$. This large momentum transfer is known as a hard scale which can also be defined as the transverse energy of a jet or the mass of a heavy quark[8]. The produce of the direct photons in two type processes: i- Quantum chromodynamics Compton sub process gq $\rightarrow \gamma q$ and ii-sub processq $\bar{q} \rightarrow$ $\gamma$ g. In this paper, we can study and calculate the Gama rate produced for three quarks system interaction and therefore one can be described the Quantum chromodynamics completely depending on the our results .

\section{2- Theory}

Quantum chromodynamics based on quantum field theory explained and describe the strong force between quarks and gluons particles with a color charge through the coupling constant.To investigate the photon's rate as a potential signature of quark-gluon interaction, one must convolute the rates with the space-time of the nucleus-nucleus collision. Photon's rate, $\Gamma_{\gamma}\left(\alpha_{s}, T\right)$ is the number of photons $d N$ with energy $E_{\gamma}$ emitted per unit volume per unit time in the three-momentum interval $\left[P_{\gamma}, P_{\gamma}+d^{3}\right]$, it is given in limit $E_{\gamma} \gg T$ by[9].

$\Gamma_{\gamma}\left(\alpha_{s}, T\right)=\frac{n_{c} C_{F}}{8 \pi^{2}}\left(\sum_{f} e_{f}^{2}\right) \alpha \alpha_{s} \operatorname{Ln}\left(\frac{2.912 E_{\gamma}}{4 \pi \alpha_{s} T}\right) T^{2} e^{-\frac{E_{\gamma}}{T}}$

Where $n_{c}$ is the colour quantum number,$e_{f}$ is the electric charge of the quark, $\alpha$ is the QED coupling constant $\alpha=\frac{e^{2}}{4 \pi \varepsilon_{o} \hbar c} \approx 1 / 137$, and $C_{F}$ is the corresponding Casimiroperator of the fundamental representation of color $\mathrm{SU}(3)$. The $\mathrm{C}_{\mathrm{F}}$ is given by [10].

$C_{F}=\frac{n_{c}{ }^{2}-1}{2 n_{c}}$ 
The effective coupling constant of the QCD depending on the $\mu^{2}$ scale by renormalized equation [11].

$\mu^{2} \frac{\partial \alpha_{S}}{\partial \mu^{2}}=\beta\left(\alpha_{S}\right)$

Where $\beta\left(\alpha_{s}\right)$ is the function for coupling constant $\alpha_{s}=\frac{g_{s}^{2}}{4 \pi}$ and given by [12].

$\beta\left(\alpha_{s}\right)=-\alpha_{s}\left[\beta_{0} \frac{a_{s}}{4 \pi}+\beta_{1}\left(\frac{a_{s}}{4 \pi}\right)^{2}+\beta_{2}\left(\frac{a_{s}}{4 \pi}\right)^{3}+\beta_{3}\left(\frac{a_{s}}{4 \pi}\right)^{4}+\cdots \ldots . ..\right]$

For least order solving, we inserting only the first term of the power expansion of $\beta\left(\alpha_{s}\right)$ in Eq.(3) ,and integrating both side results .

$\frac{1}{a_{s}\left(\mu^{2}\right)}=\frac{1}{a_{s}\left(\mu_{0}^{2}\right)}-\frac{\beta_{0}}{4 \pi} \ln \frac{\mu^{2}}{\mu_{0}^{2}}$

On the other hand the mass scale $\mu^{2}=\Lambda_{\mathrm{QCD}}{ }^{2}$ is also generated and $\alpha$ s becomes too large for the perturbative approach to be valid.

$\frac{1}{a_{S}\left(\mu^{2}=\Lambda_{Q C D}{ }^{2}\right)}=0 \rightarrow a_{S}\left(\Lambda_{Q C D}{ }^{2}\right)$

Choosing $\Lambda_{\mathrm{QCD}}=\mu_{\mathrm{o}}$, where $\Lambda_{\mathrm{QCD}}$ is a scale parameter of QCD, and Eq. (6) can rewrite as,

$\alpha_{S}\left(\mu^{2}\right)=\frac{4 \pi}{\beta_{0} \ln \frac{\mu^{2}}{\Lambda_{Q C D}{ }^{2}}}$

Where[13].

$\beta_{0}=\frac{11}{3} n_{c}-\frac{2}{3} n_{f}=11-\frac{2}{3} n_{f}, \beta_{1}=51-\frac{19}{3} n_{f}, \beta_{2}=2857-\frac{5033}{9} n_{f}+\frac{325}{27} n_{f}^{2}$

Where $n_{c}$ is the number of colors and $n_{f}$ is the number of quark flavors.

Inserting Eq.(8) in Eq.(7), and simply to reduced to.

$\alpha_{s}(\mu)=\frac{6 \pi}{\left(33-2 n_{f}\right) \ln \frac{\mu}{\Lambda_{Q C D}}}$

For thermal energy $\Lambda_{\mathrm{QCD}}=\mathrm{T}_{\mathrm{c}}$ and $\mu \approx 8 \mathrm{~T}$ for high energy collision ,Eq.(9) may be written by.

$\alpha_{S}(T)=\frac{6 \pi}{\left(33-2 n_{f}\right) \ln \left(\frac{8 T}{T_{C}}\right)}$

where $T_{C}$ is the transition temperature characterizes the critical point in which quarks and gluons become confined . substituting Eq.(10) in Eq.(1),we get :

$\Gamma_{\gamma}\left(\alpha_{s}, T\right)=\frac{3\left(n_{c}{ }^{2}-1\right)}{8 \pi}\left(\sum_{f} e_{f}^{2}\right) \frac{a}{\left(33-2 n_{f}\right) \operatorname{Ln}\left(\frac{8 T}{T_{c}}\right)} \ln \left(\frac{2.912 E_{\gamma}}{4 \pi \alpha_{s} T}\right) T^{2} e^{-\frac{E_{\gamma}}{T}}$

\section{3- Results}

In the systems of quark-gluon, one of the most important parameter for the studies and calculated the photon's yield rate is the strength coupling constant $\alpha_{\mathrm{s}}(\mathrm{T})$. It is not constant but varies with scale .This is analogous to the fine structure constant $\alpha$, that used to represent the coupling strength in QED. It can be evaluated theoretically using the Eq.(10) by using Matlab program version 7 for the thermal energy $T=150$ to $350 \mathrm{MeV}$. To calculate the strength coupling constant $\alpha_{\mathrm{s}}(\mathrm{T})$ involved estimated the values of flavors for quarks in the quark-gluon system using expression $\mathrm{n}_{\mathrm{f}}=\sum_{\mathrm{i}=1}^{6} \mathrm{n}_{\mathrm{fi}}$. Inserting the values of flavors for quarks $n_{f}=3$ for $\mathrm{u}-\mathrm{d}$ quarks system and $\mathrm{n}_{\mathrm{f}}=5$ for $\mathrm{u}-\overline{\mathrm{c}}$ quarks, with critical temperature $\mathrm{T}_{\mathrm{c}}=144 \mathrm{MeV}$ in Eq.(10),we can evaluated the values of coupling constant $\alpha_{\mathrm{s}}(\mathrm{T})$ for $\mathrm{u}-\mathrm{d}$, and for $\mathrm{u}-\overline{\mathrm{c}}$ quarks system, results are shown in Table (1).

Table(1):Results of strength coupling constant $\alpha_{\mathrm{s}}(\mathrm{T}) \mathrm{GeV}$ for $u-d$, and for $u-\bar{c}$ quark

\begin{tabular}{|c|c|c|}
\hline \multirow{2}{*}{$\mathrm{T}(\mathrm{MeV})$} & \multicolumn{2}{|c|}{$\alpha_{\mathrm{s}}(\mathrm{T}) \mathrm{GeV}$} \\
\cline { 2 - 3 } & $\mathrm{n}_{\mathrm{f}}=3$ & $\mathrm{n}_{\mathrm{f}}=5$ \\
\hline 150 & $0.3292 \mathrm{Ge}$ & 0.3865 \\
\hline 175 & 0.3069 & 0.3603 \\
\hline 200 & 0.2899 & 0.3403 \\
\hline 225 & 0.2764 & 0.3244 \\
\hline 250 & 0.2653 & 0.3114 \\
\hline 275 & 0.2560 & 0.3005 \\
\hline 300 & 0.2481 & 0.2912 \\
\hline 325 & 0.2412 & 0.2832 \\
\hline 350 & 0.2352 & 0.2761 \\
\hline
\end{tabular}

To calculate the rate of photon's yield for both systems ,we must use the electric charge of the quark with flavor (f) by $\sum_{f} e_{f}^{2}$ in units of the electron charge e. Next, we can calculate the rate of photon's yield for both systems ( $u d \rightarrow \gamma g$ and $u \bar{c} \rightarrow \gamma g$ ) by inserting the values of $\alpha_{\mathrm{s}}(\mathrm{T}) \mathrm{GeV}, \sum_{f} e_{f}^{2}, E_{\gamma}=0.5-5 \mathrm{GeV}$ [12], the colour quantum number $n_{c}=3$ in Eq.(11) with the quantum electromagnetic coupling constant $\alpha \approx 1 / 137$, and the calculated Casimiro operator $C_{F}$ from Eq.(2), results of the photon's yield rate has been summarized in Table (2) and figure (1)for $u d \rightarrow \gamma g$, and Table (3) and figure (2)for $u \bar{c} \rightarrow \gamma g$ systems.

Table(2):Results of the photon's yields rate for $u d \rightarrow \gamma g$ system

\begin{tabular}{|c|c|c|c|c|}
\hline \multirow{2}{*}{$E_{\gamma}(\mathrm{GeV})$} & \multicolumn{4}{|c|}{$\Gamma_{\gamma}\left(\alpha_{s}, T\right) \frac{1}{\mathrm{GeV}^{2} f m^{4}}$} \\
\cline { 2 - 5 } & $\mathrm{T}=150 \mathrm{MeV}$ & $\mathrm{T}=200 \mathrm{MeV}$ & $\mathrm{T}=250 \mathrm{MeV}$ & $\mathrm{T}=300 \mathrm{MeV}$ \\
\hline & $\alpha_{s}(T)=0.3292 \mathrm{GeV}$ & $\alpha_{s}(T)=0.2899 \mathrm{GeV}$ & $\alpha_{s}(T)=2653 \mathrm{GeV}$ & $\alpha_{s}(T)=0.2461 \mathrm{GeV}$ \\
\hline 1 & $2.99 \times 10^{-9}$ & $2.22 \times 10^{-8}$ & $7.80 \times 10^{-8}$ & $1.85 \times 10^{-7}$ \\
\hline 1.5 & $1,34 \times 10^{-10}$ & $2.35 \times 10^{-9}$ & $1.39 \times 10^{-8}$ & $4.74 \times 10^{-8}$ \\
\hline 2 & $5.51 \times 10^{-12}$ & $2.24 \times 10^{-10}$ & $2.22 \times 10^{-9}$ & $1.06 \times 10^{-8}$ \\
\hline 2.5 & $2.16 \times 10^{-13}$ & $2.04 \times 10^{-11}$ & $3.35 \times 10^{-10}$ & $2.25 \times 10^{-9}$ \\
\hline 3 & $8.29 \times 10^{-15}$ & $1.81 \times 10^{-12}$ & $4.91 \times 10^{-11}$ & $4.63 \times 10^{-10}$ \\
\hline
\end{tabular}




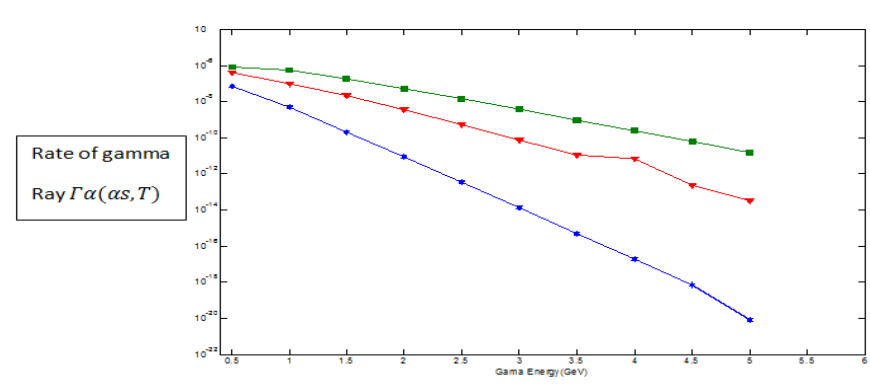

Figure (1): Rate of photon's yield for $u d \rightarrow \gamma g$ system.

The Gama energy $E_{\gamma}$ and temperatures T dependence of the rate of photon production are presented for three temperatures $\mathrm{T}=150 \mathrm{MeV}, \mathrm{T}=250 \mathrm{MeV}$, and $\mathrm{T}=350 \mathrm{MeV}$.

Table(3):Results of the photon's yield rate for $u \bar{c} \rightarrow \gamma g$ system

\begin{tabular}{|c|c|c|c|c|}
\hline \multirow{2}{*}{$E_{\gamma}(\mathrm{GeV})$} & \multicolumn{4}{|c|}{$\Gamma_{\gamma}\left(\alpha_{s}, T\right) \frac{1}{\mathrm{GeV}^{2} f m^{4}}$} \\
\cline { 2 - 5 } & $\mathrm{T}=150 \mathrm{MeV}$ & $\mathrm{T}=200 \mathrm{MeV}$ & $\mathrm{T}=250 \mathrm{MeV}$ & $\mathrm{T}=300 \mathrm{MeV}$ \\
\hline & $\alpha_{s}(T)=0.3865 \mathrm{GeV}$ & $\alpha_{s}(T)=0.3403 \mathrm{GeV}$ & $\alpha_{s}(T)=0.3114 \mathrm{GeV}$ & $\alpha_{s}(T)=0.2912 \mathrm{GeV}$ \\
\hline 1 & $5.04 \times 10^{-9}$ & $3.69 \times 10^{-8}$ & $1.27 \times 10^{-7}$ & $2.99 \times 10^{-7}$ \\
\hline 1.5 & $2.32 \times 10^{-10}$ & $4.03 \times 10^{-9}$ & $2.37 \times 10^{-8}$ & $8.01 \times 10^{-8}$ \\
\hline 2 & $9.62 \times 10^{-12}$ & $3.89 \times 10^{-10}$ & $3.82 \times 10^{-9}$ & $1.82 \times 10^{-8}$ \\
\hline 2.5 & $3.80 \times 10^{-13}$ & $3.57 \times 10^{-11}$ & $5.82 \times 10^{-10}$ & $3.91 \times 10^{-9}$ \\
\hline 3 & $1.46 \times 10^{-14}$ & $3.18 \times 10^{-12}$ & $8.60 \times 10^{-11}$ & $8.11 \times 10^{-10}$ \\
\hline
\end{tabular}

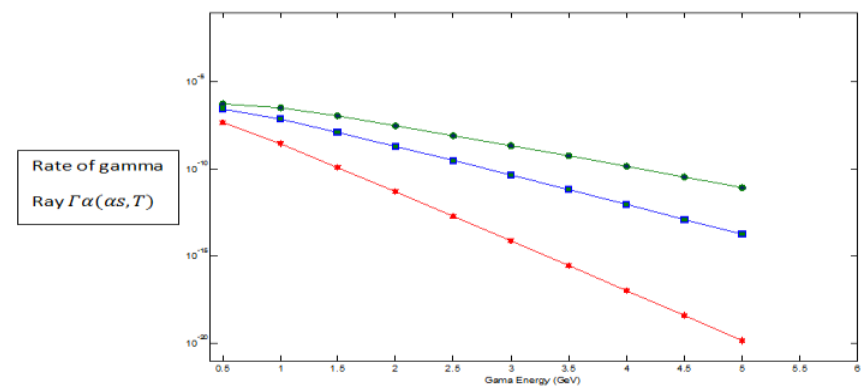

Figure (2): Rate of photon's yield for $u \bar{c} \rightarrow \gamma g$ system. The Gama energy $E_{\gamma}$ and temperatures T dependence of the rate of photon production are presented for three temperatures $\mathrm{T}=150 \mathrm{MeV}, \mathrm{T}=250 \mathrm{MeV}$, and $\mathrm{T}=300 \mathrm{MeV}$.

\section{4- Discussion}

Depending on the QCD theory, we exhibit many features for the high energy physics, which will be discussed to somewhat more detail for quark-gluon interaction. One of these the coupling constant becomes scale dependent that's shown from Eq.(10), it is clear that for small energy scales $\mathrm{T}=150 \mathrm{MeV}$, the coupling is strong $\left[\alpha_{s}(T)=0.3292 \mathrm{GeV}\right.$ for $u-d$ system , and $\alpha_{s}(T)=0.3865 \mathrm{GeV}$ for $u-\bar{c}$ system] and the $\alpha_{s}(T)$ approaches to 0 when Tincreases to infinity. This indicate that when the two quarks are close to each other the strong force is relatively weak or the quantum chromodynamics at small length scale (high energy $\mathrm{T}=$ $300 \mathrm{~V}$ ) leads to small of the coupling strength. This means that the strong force is weak if the quarks are close to each other and called asymptotic freedom. On the other hand, when two quarks move farther apart the force becomes stronger and the strength of the long distance is called confinement. Data results in Tables(2and3) and figures (1-2) show that the rates of photons are increased when decreases $\alpha_{s}(T)$ with increasing $\mathrm{T}$ and vice versa. This because of at the short distance between the quark and anti-quark pair, have the strength of the coupling is small. In particular the coupling strength of the strong interaction becomes weak as the $T$ is larger in a process (or vice versa) and quarks become free at high energy and called asymptotic freedom. Also at a long distance limit that enough to create a new quark and anti-quark pair, the binding of the original quark and anti-quark pair breaks and each quark and anti-quark are bound with the new quark and anti-quark into new two quark and anti-quark pairs that is view in figure(3). Short distance between the quark and other quark or anti quark pair has small strength of the coupling, and the coupling strength of the strong interaction becomes weak as the $T$ is larger in a process (or vice versa) and quarks become free at high energy and called asymptotic freedom.

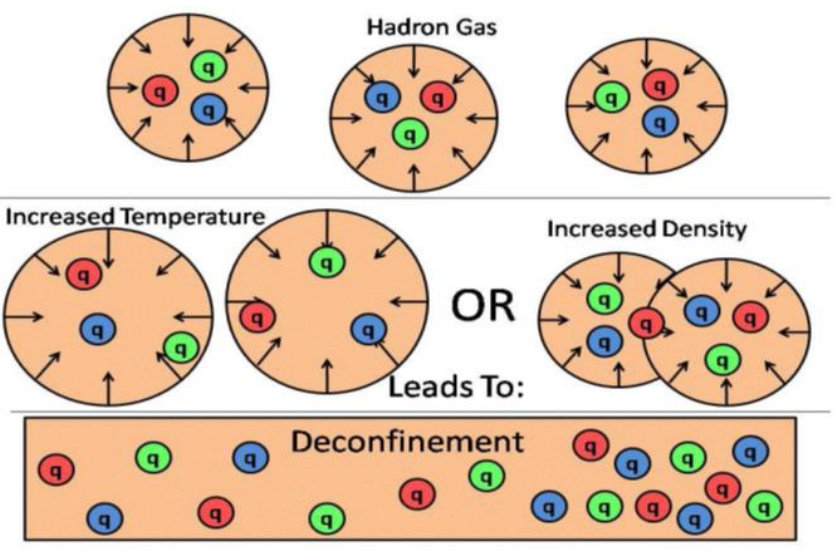

Figure 3: Deconfinement representation [5]. 
Rates of photons are increased for weak quark-gluon interaction due to short distance and asymptotic behavior, and the rate of photons are decreases at long distance and called confinement. On the other hand The rate of photons process in the $u d \rightarrow \gamma g$, and $u \bar{c} \rightarrow \gamma g$ systems can be traced back to the factor $T^{2} e^{-\frac{E_{\gamma}}{T}}$ that favors the production of photons. Figure 1 and figure 2 show that the rate depending on the energy of gamma $E_{\gamma}$ for temperatures, $\mathrm{T}=150-300 \mathrm{MeV}$ respectively , and decay is too slow.

\section{5- Conclusions}

We have seen that in strong interaction of the quarkgluon system dependent on our understanding and description of quantum cromodynamic. The force between a quarks at short distances arises due to gluon exchange to rearrange the colour structure of the final state. We conclude that the coupling constant of the QCD in the high-temperature $\mathrm{T}=300 \mathrm{MeV}$ is small and quarks are close to each other .It is asymptotic freedom behavior of quarks. The coupling varies as a function of the temperature $\mathrm{T}$. We find that the strength coupling constant influence strongly on the photon's rate yield depending on the temperature. The photon's rate is inversely proportional to the temperature at asymptotic separation and the resolution energy of gamma, in the other word, the rates of photons are increased when decrease $\alpha_{\mathrm{s}}(\mathrm{T})$ with increasing $\mathrm{T}$ and vice versa. Also we conclusion that the force become strong when two quarks move farther and called confinement, and the rate of photons are decreased.

\section{6- References}

1-Yun Guo,2009" Quarkonium States in an Anisotropic Quark-Gluon Plasma" zurErlangung des Doktorgrades der Naturwissenschaften vorgelegtbeimFachbereichPhysik der Johann Wolfgang Goethe-Universit" at Frankfurt.

2-Nicusor T.2002"The colour of gluon interactions Studies of Quantum Chromodynamics in soft and hard processes" $\mathrm{Ph}$ D. Thesis ,Acta Universitatis Upsaliensis Uppsala.

3-Kristin Fanebust Hetland,2005"Production of strange particles in lead-lead interactions at $158 \mathrm{~A} \mathrm{GeV/c"}$ $\mathrm{PhD}$ Thesis, Universities Bergen, Bergen ,Norway. 4-Myron W. Evans " Quark gluon model in the evans unified field theory" Book, Chapter 12, Alpha foundation's institute for advance study (AIAS). (emyrone@oal.com,

www.aias.us, www.atomicprecision.com)].

5-Stephen Baumgart, 2009"A Study of open charm production in heavy ion collisions of center-ofmass energy $200 \mathrm{GeV}$ per nucleon"PhDThesis ,Yale University.

6-M.M. Aggarwal et al., 2000 (WA98 Collaboration), "Direct photon production in $158 \mathrm{~A} \mathrm{GeV}$ $208 \mathrm{~Pb}+208 \mathrm{~Pb}$ Collisions", nucl-ex/0006007, (June).

7-Stephen Andrew Bull, 2005"Strange Particle and Antiparticle Production in Proton-Beryllium Interactions at $40 \mathrm{GeV} / c$ at the CERN NA57 Experiment" PhD Thesis, University of Birmingham.

8-Sarah Boutle,2009,"Beauty in photoproduction at HERA II with the ZEUS detector " PhD thesis ,University College London, London.

9-Christian Klein-Bösing,2004"Production of Neutral pions and direct photons in ultra-relativistic $\mathrm{Au}+\mathrm{Au} \quad$ collisions" $\mathrm{PhD}$ Thesis, Naturwissenschaften in Fachbereich Physic and Mathematics WestfälischenWilhelms-University Münster.

10-Maria E. T. Y.2001," Proton-Proton Scattering at two loops", Ph.D. thesis, Durham University, Durham.

11-Jiri Kvita ,2009"Measurement of Tev with the DO experiment at fermilab" PhD Thesis ,Charles University .

12-Takuma Horaguchi,2006"Prompt photon production in proton-proton collisions at $\mathrm{p}_{\mathrm{s}}=200 \mathrm{GeV}^{\prime \prime} \mathrm{PhD}$ Thesis, Tokyo Institute of Technology.

13- Philipp Tim Reuter, 2005 "A General effective action for quark matter and its application to color superconductivity" $\mathrm{PhD}$ Thesis University of Frankfurt Frankfurt.

14-Takuma Horaguchi, 2006"Prompt photon production in proton-proton collisions at ps $=$ 200GeV"PhD Thesis ,Tokyo Institute of Technology, Tokyo. 